\title{
ANHANG
}

\section{„Vielheit und Einheit der Germanistik weltweit“". XII. Kongress der Internationalen Vereinigung für Germanistik. Warszawa, 30.7.-7.8.2010}

Vom 30. Juli bis 7. August 2010 trafen sich über 1.500 Germanisten in Warschau zum XII. Kongress der Internationalen Vereinigung für Germanistik, der unter dem Motto ,Vielheit und Einheit der Germanistik weltweit" stand. Dass Polen in Paris 2005 als Gastgeber des nächsten Kongresses ausgewählt wurde, unterstreicht, welch großer Rang der polnischen Germanistik international zugesprochen wird. Der in 60 Sektionen organisierte Warschauer Kongress dokumentierte die Vielfalt der gegenwärtigen internationalen germanistischen Forschung, die ihren medialen und disziplinären Rahmen oftmals weit überschreitet, wie u.a. Sektionen zum Verhältnis von Literatur und anderen Medien (Film, Musik und bildende Kunst) und anderen Disziplinen (Geschichte) zeigten. An dieser Stelle können nur einige Schwerpunkte aus dieser Vielfalt hervorgehoben werden. $\mathrm{Zu}$ diesen zählten zweifellos die recht modischen Themen Interkulturalität und Transnationalität, die, sich dabei teilweise überlagernd, im Zentrum mehrerer Sektionen und Panels standen. Beispielsweise beschäftigten sich die Referate und Diskussionen in den literaturwissenschaftlichen Sektionen, in denen wiederum die Nachkriegs- und die Gegenwartsliteratur überwogen, anhand der Literatur deutsch-türkischer Schriftsteller, polnischer, serbischer, kroatischer Migranten oder rumäniendeutscher Schriftsteller häufig mit Fragen der multikulturellen Identität und der Er- zählung von interkulturellen Familiengeschichten. Ein weiteres, häufig diskutiertes Thema, oftmals mit dem interkulturellen Erzählen verknüpft, war das Verhältnis von Literatur und Erinnerung. Fragen nach dem genealogischem Gedächtnis, nach der Beziehung zwischen Geschlecht und Erinnerung, nach Narrativen postnationaler, traumatischer und inkorporierter Erinnerung standen hier im Vordergrund, wobei auf das ,,postmemory"-Konzept von Marianne Hirsch ebenso wie auf Friederike Eiglers Arbeit Gedächtnis und Geschichte im Generationenroman nach der Wende rekurriert wurde. Einige wenige Romane wurden immer wieder zum Gegenstand von Referaten. $\mathrm{Zu}$ ihnen gehörten Tanja Dückers' Himmelskörper, Ilija Trojanows Der Weltensammler, Zafer Şenocaks Gefährliche Verwandtschaft, Doran Rabinović' Ohnehin, Katrin Schmidts Die Gunnar-Lennefsen-Expedition. Die interkulturelle Positionierung der germanistischen Forschung dokumentierten darüber hinaus Sektionen zur deutschsprachigen Kultur in Lateinamerika, zur Niederlandistik und „Luxemburgistik im Spannungsfeld von Mehrsprachigkeit, Regionalität, Nationalität und Internationalität“, zum Indien-Bild oder zum Europadiskurs in der deutschen Literatur. Welche Schwerpunke es neben diesem übergreifenden Thema der Interkulturalität in Sprache und Literatur gab, zeigen die folgenden ausgewählten Sektionsberichte. 
Sektion 3: „Literatur, Kunst und Musik im Kontext Deutsch als Fremdund Zweitsprache“ (Leitung: Camilla Badstübner-Kizik)

Am ersten Tag dieser Sitzung standen Aspekte der Literaturdidaktik im Kontext DaF/DaZ im Mittelpunkt der Erwägungen, die Beiträge hatten sowohl konzeptionellen als auch exemplarischen Charakter. Claus AltMAYER (Herder-Institut, Universität Leipzig) eröffnete mit dem Referat „Der Beitrag literarischer Texte zum kulturellen Lernen in Deutsch als Fremdsprache", in dem er neuere Theoriediskussionen in den Literatur- und Kulturwissenschaften für die Beschäftigung mit literarischen Texten im Kontext $\mathrm{DaF} / \mathrm{DaZ}$ fruchtbar zu machen versuchte. Sein Anliegen war es, auf der Grundlage von aktuellen Theoriemodellen ein Konzept von „,kulturellem Lernen“ zu entwickeln und zu zeigen, wie literarische Texte aufgrund ihrer spezifischen ,Literarizität ${ }^{*} \mathrm{zu}$ kulturellen Lernprozessen beitragen können. Es folgten drei Beiträge zur Kinder- und Jugendliteratur. MERLE JUNG (Tallinn) sprach zu ,Autobiografische(n) Texte(n) deutscher Jugendlicher im interkulturellen DaF-Unterricht". Anhand von Kurztexten aus dem OnlineMagazin Jetzt zeigte sie, wie kurze autobiografische Texte als Vermittler landeskundlicher Informationen dienen können, interessanter Diskussionspunkt war dabei die Frage nach deren Authentizität. Das Thema von Ellen SchUlte-BunerT (Flensburg) war „Interkulturelles Lernen mit Texten der Kinder- und Jugendliteratur". Sie diskutierte die interessante und zukunftsträchtige Frage, wie Kinder- und Jugendliteratur (vom Bilderbuch bis zum Jugendroman) interkulturelles Lernen initiieren und auf das Leben in multikulturellen Gesellschaften vorbereiten bzw. dieses begleiten können. ALDONA SOPA-
TA (Poznań) widmete sich „Kinderbüchern im frühen Fremdsprachenunterricht" und situierte das Thema im neurophysiologischen, psychologischen und kognitiven Kontext. Ihr Ziel war, Vorschläge für eine effizientere Arbeit mit Kinderbüchern zu unterbreiten, die die Ergebnisse der entwicklungspsychologischen, spracherwerbs-theoretischen und fachdidaktischen Forschung besser als bisher berücksichtigen.

Es folgten vier Beiträge, in deren Mittelpunkt literarische Textgattungen standen, die bisher (noch) selten Beachtung im Kontext $\mathrm{DaF} / \mathrm{DaZ}$ finden. Gleichzeitig wurde damit deutlich, dass gerade dort ein weiter Literaturbegriff seine unbedingte Berechtigung hat. Almut Hille (Freie Universität Berlin) stellte in ihrem Referat zur Slam-Poetry die vielfältigen Potenziale dieser literarischen Gattung an konkreten Beispielen aus dem Schaffen eines der avanciertesten deutschsprachigen Slam-Poetry-Künstlers, Bas Böttcher, vor. Sie zeigte Besonderheiten des Genres und skizzierte seine intermedialen Potenziale. KISHIK LEE (Korea University, Südkorea) ging in seinem Vortrag „Leseförderung durch Unterhaltungsromane?" auf die Leseunlust koreanischer Studierender ein und präsentierte deutschsprachige Unterhaltungsromane sowie die ihre Lektüre begleitenden didaktischen Maßnahmen (Lesetagebuch, Leseprotokolle) als einen möglichen Ausweg aus der Lesekrise. MANJIRI PARANJAPE (University of Pune, Indien) machte in ihrem Beitrag zu „Übersetzungen von Bühnentexten aus didaktischer Perspektive im Rahmen des Germanistikstudiums in Indien" deutlich, dass diese nicht nur zur Verbesserung der Sprachkompetenz beitragen, sondern auch auf besonders effektive Art eine bessere Sensibilisierung für Eigenes und Fremdes ermög- 
lichen können. Den ersten Sektionstag beschlossen SYLwIA ADAMCZAK-KRYSZTOFowicz (Poznań), AnTJe Stork (Phillips-Universität Marburg) und PAWEE RYBSZLEGER (Poznań) mit dem Thema „Hörbücher: Möglichkeiten des Einsatzes im DaF-Unterricht", auch dies eine didaktisch bisher noch stark unterschätzte Textsorte. Die drei Referenten stellten eine mögliche Klassifikation von Hörbüchern vor und diskutierten auf der Grundlage von empirischen Daten verschiedene Möglichkeiten, diese als intermediale Textkombinationen für eine integrativen Hörverstehensentwicklung einzusetzen.

Die Beiträge des ersten Sitzungstages waren vom Niveau und wissenschaftlichen Anspruch etwas uneinheitlich, aber durchweg sehr inspirierend, sie wurden im Anschluss jeweils lebhaft diskutiert und insgesamt von den Teilnehmern als Bereicherung erfahren. Deutlich wurde u. a. der Bedarf an weiterer empirischer Forschung im Bereich der Literaturdidaktik.

Am zweiten Sitzungstag standen mit insgesamt vier Beiträgen intermediale Kombinationen (Sprache-Musik-Bewegung) im Mittelpunkt, die Spannbreite an literatur- und kulturdidaktisch interessanten Textgattungen wurde damit noch erweitert. ANNEDORIS BAUMANN (Universität Nikosia, Zypern) zeigte in ihrem Beitrag „Szenische Interpretation von Musik im Fremdsprachenunterricht" am Beispiel von Brechts Dreigroschenoper, welchen günstigen Einfluss musikpädagogische Verfahren wie die szenische Interpretation auf Effektivität, Themen- und Lernerzentrierung des Fremdsprachenerwerbs nehmen können. Ausgehend von Erfahrungen aus dem bilingualen Unterricht zeigte sie Möglichkeiten, wie dieses Verfahren auch im DaF-Unterricht genutzt werden kann. WALTRAUT BRIGITTE MAYR (Universität Malaysia) stellte ein geplantes Projekt vor, das über die Beschäftigung mit deutschsprachigen Liedern aus verschiedenen Epochen Einfluss auf das in Malaysia herrschende ambivalente bis negative Deutschlandbild nehmen will. Sie präsentierte theoretische Überlegungen aus dem Bereich der einschlägigen Forschung und machte auf die Möglichkeiten aufmerksam, die linguistische und kulturelle Vergleiche von deutschsprachigem und malayischem Liedgut bieten können. REINHOLD UTRI (Warschau) demonstrierte in seinem praktisch angelegten Beitrag „Deutsches Liedgut als relevantes kulturelles Element im modernen DaF-Unterricht", wie Musik in einem holistisch angelegten glottodidaktischen Kontext sprach- und motivationsfördernd wirken sowie zu interkulturellem Lernen anregen kann. Der ebenfalls praktisch angelegte abschließende Beitrag von Magdalena ŻYŁKO-GROELE (Katowice) zeigte am Beispiel eines fortlaufenden Unterrichtsprojektes zu Joseph Haydn, wie sich Kulturwissen in den Fremdsprachenunterricht integrieren lässt. Die Diskussion der wiederum sehr unterschiedlichen Beiträge des zweiten Tages verlief in kleinem Kreis, die Aussagen der einzelnen Beiträger brachten gerade hier nicht sehr viel Neues. Zu bedauern ist, dass viele literatur- und kulturdidaktische Beiträge, die die Diskussion in Sektion 3 bereichert hätten, zeitgleich in anderen Sektionen präsentiert wurden, eine inhaltliche Koordination, eine bessere Gewichtung und mitunter deutlichere Profilierung der einzelnen Sektionen und Sektionsthemen untereinander wäre sicher sinnvoll gewesen.

Camilla Badstübner-Kizik, Poznań 
Informationen und Berichte

Sektion 4: Sprache in der Literatur (Leitung: Anne Betten, Hans Höller, Rita Svandrlik)

Der Sektionstitel mag auf den ersten Blick für Irritation sorgen - dass Sprache das Material der Literatur ist, scheint offensichtlich, genauso wie die Tatsache, dass der Stoff der Literatur über die Sprache vermittelbar ist. Die Vieldeutigkeit des Wortes ,Sprache“ galt allerdings in diesem Fall als Programm, welches in einem produktiven Spannungsverhältnis zwischen linguistischen und literaturwissenschaftlichen Fragestellungen changierte. Eine solche unorthodoxe Zusammenführung von Sprach- und Literaturwissenschaft innerhalb einer Sektion verdankte man österreichischen Germanisten: Ursprünglich sollte die Sektion von Anne Betten aus Salzburg (Sprachwissenschaft) und Wendelin Schmidt-Dengler (Literaturwissenschaft) geleitet werden. SchmidtDengler ist im Herbst 2008 nach kurzer Krankheit gestorben, was die österreichische (um nicht zu sagen: internationale) Germanistik in tiefe Trauer versetzt hat. Die Co-Leitung wurde von Hans Höller und Rita Svandrlik übernommen, wodurch die literaturwissenschaftliche Fraktion in der Sektion eine Stärkung bekommen hat. Einer durchaus gelungenen Kooperation der Sektionsleiter verdankte die „Sprache in der Literatur" ihre thematische Offenheit und Brisanz.

Die Bandbreite der vorgenommenen Fragestellungen haben theoretisch ausgerichtete Beiträge am ersten Tag der Tagung (30.7.) eröffnet. In dieser ersten Sitzung wurden die Akzente gesetzt bzw. prägnante Aspekte für die Diskussionen der folgenden Tage genannt. Gesprochen wurde über die Zusammenhänge zwischen Introspektion und Narratologie (eine auf der Kognitivistik basierende Analyse von Thomas Eder, Wien), über die Aktuali- tät der avantgardistisch geprägten Literaturtheorie als Sprachkunst (JÜRGEN BROKOFF, Bonn), über referenzielle und polyphone Strukturen der literarischen Texte (Heinz VATER, Berlin) sowie über komplexe Verschränkungen von Disziplinen (Literaturtheorie, Semiotik, Erzählforschung, Textlinguistik, Psycholinguistik, um nur einige zu nennen), welche einen interpretatorischen Zugang zu literarischen Texten möglich machen können (RICHARD SCHRODT, Wien).

Im Laufe der Tage kamen die zuvor signalisierten theoretischen Fragestellungen in einzelnen Textanalysen oft zur Sprache, wurden auch um andere aktuelle Verfahren der literaturwissenschaftlichen wie linguistischen Textinterpretation ergänzt. So wurde u.a. Polyphonie als strukturelles Merkmal literarischer Texte thematisiert (MARIE HÉLÈne PERENNEC, Nicole Fernandez-Bravo, Paris), stilistische Strategien der Textkomposition aufgezeigt (ANGELIKA REDDER, Hamburg; Michaela ReinhaRdT, Piemont), syntaktische Charakteristika lyrischer und prosaischer Werke erörtert (SIGURD PAUl SCHEICHL, Innsbruck; HaNS HöLLER, Salzburg). Sprachexperimente wurden als ein in der österreichischen Literatur nach wie vor präsentes Interesse an der Hinterfragung der Automatismen des Sprechens und Verstehens dargestellt (ANNe BetTen, Ulrike EdER, Wien; KALINA KUPCZYŃSKA, Łódź). Eine relevante Fokussierung galt den Theatertexten der Gegenwart, deren Sprachgestaltung in ihrer Vielfalt diverse interpretatorische Herangehensweisen zulässt (Ulla FIX, Leipzig; Michaela ReinhaRdT, Piemont). Unter den analysierten Texten dominierten diejenigen österreichischer Autoren (die Interpretationsansätze galten u.a. den Werken von Thomas Bernhard, Elfriede Jelinek, Peter Handke, Friederi- 
ke Mayröcker, Marlene Streeruwitz, Kathrin Röggla). $\mathrm{Zu}$ vermissen war dabei allerdings ein Augenmerk auf weniger etablierte bzw. jüngere Vertreter österreichischer wie auch deutscher Wortkunst, die nur in einigen wenigen Beiträgen thematisiert wurden.

Einen durchaus adäquaten Schlussakzent bekamen die Diskussionen mit dem Vortrag von Neva ŠLibar (Ljubljana), in dem das Phänomen der Desautomatisierung der Wahrnehmung als ein genuines Merkmal der Literarizität dargestellt und an einigen Textbeispielen exemplifiziert wurde. Hinsichtlich der Vielzahl der von NEVA ŠLIBAR angesprochenen theoretischen Ansätze diente der Vortrag zum einen als Pointierung der aufgeworfenen Fragestellungen der Sektion, zum anderen als Anregung für das anschließende Schlussgespräch. Dieses betraf u.a. das für die Literatur- und Sprachwissenschaft relevante Moment der theoretischen Grundierung der jeweiligen interpretatorischen Zugänge an Texte, was in Anbetracht der Fülle der Theoriebildungen für beide Fächer eine Herausforderung, aber auch eine Chance bietet.

„Sprache in der Literatur“, mit 35 Vorträgen eine der größten Sektionen des Kongresses, hat sich somit gerade dank der für die Konferenzpraxis innerhalb der Germanistik ungewohnten Doppelperspektive auf die literarische Produktion als eine durchaus gelungene wissenschaftliche Leistung erwiesen. Der geplante Konferenzband wird diese Leistung dokumentieren.

Kalina Kupczyńska, Łódź

Sektion 6: Politische Romantik im 19. und 20. Jahrhundert (Leitung: Ulrich Breuer, Rehha Kamath-Raman, Grażyna Kwiecińska, Nikolaus Wegmann) Bei der Durchsicht des Tagungsprogramms fiel dem Berichterstatter auf, dass eine Sektion zur Klassik fehlte vielleicht einmalig auf einer germanistischen Tagung dieser Größenordnung. Wer sich für die Literatur um 1800 interessiert, sah sich auf thematisch ausgerichtete Sektionen wie die zum Europadiskurs (35) oder auf die RomantikSektionen (6 und 7) verwiesen. Beide griffen in die Romantik-Rezeption und bis ins 20. Jhd. aus. Insofern hätte sich vielleicht eine Zusammenlegung angeboten, doch stellte sich schnell als Vorteil der kleineren Sektion 6 heraus, dass sich in der fortlaufenden Diskussion eines begrenzten, nur wenig fluktuierenden Kreises von Referenten und Zuhörern Kreuz- und Querverbindungen zwischen den Themen und Epochen herstellten.

Konsens war, dass die Trennung von Literatur(-wissenschaft) und Politik sachlich falsch ist, sowohl weil die romantische Literatur und Philologie (überwiegend national-)politische Ziele verfolgte, als auch weil sie, allerdings von den verschiedensten Richtungen, für politische Ziele in Anspruch genommen wurde. Für das 20. Jhd. wurden etwa Carl Schmitt und Gottfried Benn für die konservative, Walter Benjamin und Heinrich Mann für die linke Romantikrezeption angeführt. In der Zeit um 1800 wurden mehrere (zusammenhängende) Verfahren der ReOrganisation des Staates ausgemacht, die die Revolutionsgefahr eindämmen sollten: Segregation und Integration sowie Legitimation und Aufwertung der alten Machthaber. Die Segregation erfolgt durch Ausgrenzung bestimmter Gruppen wie der Juden und ,Philister' bei Arnim, Brentano und der ,Christlich-deutschen Tischgesellschaft", die Integration der Restgruppe durch Strategien gesteuerter Selbstorganisation bzw. Manipulation insbesondere bei Novalis (REMIGIUS BUNIA, Berlin; Till Dembeck, Riga), die 
historische Legitimation in den sprachhistorischen Studien Fichtes und Schlegels (Michael Dusche, Erfurt). Flankiert werden diese Strategien von der Aufwertung der alten Ständeordnung durch Schlegel und Adam Müller (JoCHEN STROBEL, Marburg) sowie der traditionellen Rolle der Frau durch Kleist (Michael Mandelartz, Tokyo).

Die von Kleist in der Hermannsschlacht vorgeführte Technik, nationale Identität durch die Konstruktion von Gegensätzen herzustellen, führt Grabbe in der Hermannsschlacht mit einem skeptischen Grundton weiter (SIENTJE MAES, Leuven). Ebenso verlieren die romantischen Forderungen nach nationaler Einheit 1848 an Eindeutigkeit. Republikaner und Monarchisten, Individualisten und Kollektivisten verfechten Verfassungsentwürfe, die nur schwer unter einen Hut zu bringen sind (MARKUS HÄFNER und GERNOT UhL, Mainz).

Wie kontextabhängig die Rezeption der politischen Romantik ist, zeigte auch ANDREAS LAWATY (Hamburg). Nachdem Polen 1795 aufgeteilt und nach dem Wiener Kongress in Personalunion mit Russland vereinigt worden war, kämpfte die polnische Nationalbewegung im Rückgriff auf romantisches Gedankengut für die Wiederherstellung des Nationalstaates. Die polnische Romantik steht von Beginn an im Zeichen des Katholizismus, doch spielt auch der jüdische Messianismus eine Rolle. Erfüllte Staatlichkeit und Religion werden in die $\mathrm{Zu}$ kunft projiziert, so dass die polnische Nation den widrigen Zeitläuften zum Trotz zumindest als Projektion Bestand erhält, bis sie wieder wirklich wird.

Michael Mandelartz, Tokyo
Sektion 7: Die deutsche Romantik und die Folgen (Leitung: Min Suk Choe, Hartmut Steinecke, Walter Hinderer) HARTMUT STEINECKE (Paderborn) bezog sich in seinem einleitenden Referat unter dem Titel „Romantik. Eine deutsche Affäre?"“ auf Rüdiger Safranskis Buch über die Romantik. Diesem ginge es vor allem um den NS-Kult der deutschen Romantik. Den internationalen Kontext, den Blick von außen, die Verbindung mit anderen Künsten und Wissenschaften habe Safranski ausgeblendet, betont habe er dagegen die Reaktion auf die napoleonische Besatzung durch Görres und andere, die begannen, die deutschen mythischen Taten und das Mittelalter zu rühmen. Steinecke ging auch auf die Wirkung der deutschen Romantik ein: E.T.A. Hofmann war lange Zeit der bekannteste Autor in Paris, danach wurde er von Heine abgelöst. Thomas Mann habe in Eichendorff den typischen Romantiker gesehen, d.h. den unpolitischen Deutschen. In der Diskussion verwies Walter Hinderer auf die Präromantik, deren Verdienst es gewesen sei, eine andere Hierarchie der Sinne zu errichten: der akustische Sinn wurde von nun an wichtig, etwa bei Herder. In der Diskussion wurde Safranskis Buch als eine Mentalitätsgeschichte der Deutschen bezeichnet. KAROL SAUERLAND (Warschau), der zweite Redner, legte die unterschiedlichen Romantikauffassungen in Deutschland und Polen dar. Unter Romantik verstehe man Antiklassizisimus auf der einen Seite, politische Romantik auf der anderen. Beim ersten Begriff müsste man mit England und dem Sturm und Drang beginnen. Die polnische Romantik war anfänglich vor allem eine antiklassizistische Bewegung. Der Begriff der politischen Romantik betreffe die Zeit nach 1800. Im Referat von LEE- 


\section{Informationen und Berichte}

NA EILITTÄ (Helsinki) ging es um das Bild in der deutschen und englischen Romantik. Die Autorin beschäftigte sich vor allem mit dem Marmorbild von Eichendorff, das sie aber recht ungenau interpretierte, so dass Daniel Müller Nielaba auf die Ungewissheit hinweisen musste, ob es sich um eine Statue oder ein wirklich gesehenes Mädchen handle. Er sprach von Umschriften vom Bild zur Wirklichkeit und umgekehrt. Jemand verwies auch auf die Tendenz zur Bildlosigkeit durch den Kult der Nacht (Hymnen an die Nacht). WALTER HINDERER (Princeton) bezeichnete in seinem Vortrag „Entzauberung der Romantiknatur“ Fichte als Katalysator für die Romantik. Für wichtig hielt er auch das Systemprogramm; der Zusammenhang der Gegensätze sei in dieser Zeit aktuell gewesen, wofür er auch Schiller anführte. Entfremdung auf der einen Seite: Wackenroders Berlinger treibe es bis zur Selbstentfremdung. Hinderer zitierte auch „Wer sich selbst etwas näher kennt, wird die Menschen für Ungeheuer halten" aus dem Lovell von Tieck. Eichendorff habe solche Sichtweisen als unheimlich empfunden. Auf der anderen Seite enthält die Romantik durchaus viel Rationales. Hinderer endete mit der Forderung, von einer Dialektik der Romantik zu sprechen. Es ist eine Forderung, die jetzt immer wieder gestellt wird. GUENTER H. OESTERLE (Gießen) ging es in seinem Vortrag „Einheit in der Differenz: ,Kunstmärchen' versus ,Volksmärchen"“ vor allem darum zu zeigen, dass die Grimms keineswegs „Naturmärchen“, d.h. mündlich überlieferte Märchen geschaffen haben, $70 \%$ davon war von ihnen hinzugedichtet worden. Die Grimms gaben jedoch vor, es sei prähistorisch, präethnologisch. Sie wollten noch dazu alles als eine Sache der deutschen Mythologie auslegen.
Die Arnims und Brentanos hätten sich über eine solche Sichtweise nur lustig gemacht. Oesterle setzte an die Stelle des Aberglaubens den von Rilke geprägten Vor-Glauben. In der Diskussion wurde ihm von Märchenforschern vorgehalten, dass er die Märchen des beginnenden 19. Jhd.s zu sehr von den Feenmärchen und den schon im Mittelalter existierenden Märchen absetze. Wie ein Gegenreferat wirkte der Vortrag von DANIEL MÜLLER NiElabA (Zürich) „Epistemologie des ,Volksmärchens': ,Hans im Glück' oder: (G)Lücken des Bedeutens.“ Der Protagonist des Märchens Hans im Glück wusste bekanntlich mit dem Stück Gold, das so groß wie sein Kopf war, nichts anzufangen und vertauschte es gegen immer weniger Wertvolles. Als er nichts mehr hatte, fühlte er sich glücklich wie noch nie: „,So glücklich wie ich', rief er aus, ,gibt es keinen Menschen unter der Sonne." Mit leichtem Herzen und frei von aller Last sprang er nun fort, bis er daheim bei seiner Mutter war." Der Referent spielte mit den Begriffen ,narratio und ,doxa', was eine gute Ergänzung zu Oesterles Ausführungen darstellte, bei dem es ja auch um das Geschriebene und Gesprochene ging. Unter den weiteren Referaten ragte das von BARBARA SUROWSKA (Warschau) „Von Tieck zu Pirandello. Experimente mit der Theatertechnik" heraus, das vier Theaterstücke von Tieck, die gegen jede Theaterkonvention ,rebellierten“, und Sechs Personen suchen einen Autor zum Vorwurf hatten. Tieck versuchte, dem Heterogenen, das das eigentlich Ganze darstellt, gerecht zu werden. Es kommt zu einem ständigen Rollenwechsel bis hin zum Wechsel von Publikum und Schauspieler. Das Publikum will keinen Apollo mehr sehen, der von sich selbst sagt, dass er doch auch nur ein Spieler sei, kein 
wirklicher Gott. Tieck erwägt auch die Möglichkeit des Weitersprechens, d.h. es wird kein Ende gefunden, es kann einfach immer weitergehen. Das Theater müsse mit sich selbst scherzen. Pirandello meint dagegen, dass das Theater sich selbst reflektieren müsse. Es wäre im nächsten Schritt theoretisch anzugehen: Wie lässt sich die Grenze zwischen $\mathrm{Zu}$ schauer und Theater überwinden? Theater als Erziehungshilfe (etwa für die Väter, die in eine Ehe nicht einwilligen wollen), indem Theater im Theater gespielt wird. Die steten Veränderungen von Theaterkonventionen unter dem Einfluss historischer Veränderungen (im konkreten Fall war es die Französische Revolution), die Abhängigkeiten zwischen Autor, Spielleiter, Schauspieler, Zuschauer werden von Tieck als zum Theater zugehörig reflektiert. Er bedenkt auch die Möglichkeit, dass der Zuschauer zum Spieler wird. Was Tieck hier spielerisch anspricht, erinnere an das heutige Fernsehen und an die zunehmende Rolle von Darstellern und besonders Darstellerinnen, die völlig unprofessionell auf die Bühne, ins Bild treten. Eine gute Ergänzung hierzu war der Vortrag von ANASTANCA TABARASI über Baggesens Stück Der unvollendete Faust von 1810. Vieles erinnerte an Tiecks Theatersatiren. Baggesen hatte an das Faust-Fragment von 1790 angeknüpft. Hier werde auch Fichtes Ich-Auffassung, die Reinhold, ein Freund Baggesens, zu konkretisieren suchte, ironisch dargestellt. Min SuK ChOES (Seoul) Vortrag ,Romantik - eine deutsche Affäre auch in Korea? Rezeption der deutschen Romantik in Korea“ musste verlesen werden. Er war als Ergänzung zu den beiden einleitenden Referaten gedacht. Die deutsche Romantik wurde in Korea nach der Öffnung des Landes in den 20er Jahren des 20. Jhd.s rezipiert, den Übersetzungen lag oft nicht die Originalsprache zugrunde. Sie spielte eine ähnliche Rolle wie hundert Jahre zuvor in Polen, d.h. sie diente der nationalen Erhaltung. Es folgte ein glänzendes Referat von MichaEL WeITZ (Athen) über die Marionette, wobei der Referent das Marionettentheater von Kleist wegließ. Er nannte Tiecks Lovell, Schlegels Lucinde, Hoffmanns Sandmann und Büchners Danton. Es ging ihm u.a. darum, dass der Enthusiast sich wie eine Marionette benehme und dass wir es immer mit einer Entstellung des Körpers zu tun hätten (es kann sich auch um eine geistige Entstellung handeln). Alles geht marionettenhaft vor sich und der Entstellungen gibt es viele. Weitz verwarf allerdings den Enthusiasmus zu enthusiastisch, wie in der Diskussion bemerkt wurde. Alle bedauerten, dass es zwei Romantiksektionen gab, denn sicherlich wurde oft über das Gleiche debattiert.

Karol Sauerland, Warszawa

Sektion 20: „Nationale Erinnerungskulturen im Zeitalter der Globalisierung" (Leitung: Janusz Golec, Maria Kłańska, Irmela von der Lühe)

Die Sektion tagte in der Aula des Staszic-Palais, das zur Akademie der Wissenschaften (PAN) in Warschau gehört. Es wurden 21 Vorträge gehalten, und zwar je 6 von deutschen und polnischen Teilnehmern, 3 von den Referenten aus Kroatien, 2 aus der Ukraine und jeweils einer aus Frankreich, Irland, Japan und den USA. Da die Sektion durch die $\mathrm{Zu}$ sammenlegung zweier Sektionen, der genannten und der Sektion zu Galizien und Bukowina entstanden war, betraf der erste Themenkomplex die kollektive kulturelle Erinnerung dieser Gebiete, z.B. befasste er sich mit der unterschiedlichen kulturellen Erinnerung und ihrer Bewer- 
tung von Seiten der ehemaligen Völker Galiziens und der Bukowina, mit der Problematik der „Dichtung und Wahrheit" in autobiographischen Erinnerungen und in epischer Prosa sowie der Relativität der Geschichtsschreibung.

Weitere Vorträge bezogen sich auf die Shoah und die untergegangene ostjüdische Welt, so beschäftigte sich z. B. ein Vortrag mit der Geschichte der jüdischen Gemeinschaft in der polnischen Stadt Lublin anhand der Studie des jüdischen, von den Nazis umgebrachten Gelehrten Majer Balaban oder mit der Erinnerung an die Shoah im Medium Film im gleichnamigen Film Claude Lanzmanns. Zum jüdischen Teil gehörte ebenfalls ein Referat zur Migrationsliteratur, und zwar über die jüdischen Lebensgeschichten des aus Russland ausgewanderten Vladimir Vertlib. Das Thema Erinnerung im Kontext des Exils der Nazizeit behandelte ebenfalls der Vortrag über Norbert Gstrein. Bei großem Anklang der Zuhörer wurde an Paul Celans intertextuellen Dialog mit den Traditionen der deutschen Literatur erinnert. Ferner wurden verharmlosende Strategien der Darstellung des Holocaust in der westdeutschen Erzählprosa bis 1965 behandelt. Ein weiterer Vortrag zeigte die unlösbaren Dilemmata der Erinnerung an das Genozid bei einem schriftlosen Volk wie den $\mathrm{Zi}$ geunern auf, was eine lebhafte Diskussion hervorrief.

Relativ viel Platz wurde der Problematik der Erinnerung an die ehemaligen deutschen Ostgebiete/die heutigen Westgebiete Polens gewidmet, so z. B. dem Oder-Motiv in der deutschen und polnischen Literatur nach 1945. Einige Vorträge konzentrierten sich ferner auf die Erinnerungsarbeit und Mythosschaffung im Medium bezüglich der ,,verlorenen" deutschen Ostgebiete in Literatur und
Film, vor allem anhand des Themas des Schiffsuntergangs der Gustloff, sowie auf die Diskrepanzen zwischen Gedächtnis und Geschichte und die Dekonstruktion der „großen Geschichte“ durch die Dezentralisierung der Perspektive bei Grass. Das Thema wurde durch einen Vortrag über die spezifische weibliche und männliche Erinnerung fortgesetzt.

Einige Referate bezogen sich auf die kulturelle Erinnerung der Zeit der deutschen Klassik und ihre heutige Bedeutung, z. B. Die Italienische Reise Goethes vor allem im Hinblick auf die „Welt-Begriffe“ und die Opposition ,,italienisch“/,deutsch“. Eine weitere Facette der Dekonstruktion des Begriffs von der ,deutschen Kulturnation" wurde durch die Analyse von Kehlmanns Humboldt-Roman vertreten.

Einige Vorträge rückten besonders die komparatistische Sicht in den Fokus, so der Vortrag über das Irische Tagebuch Bölls sowie das dem konstruierten kulturellen Gedächtnis an das ausgerottete Volk der Pruzzen gewidmete Referat, wobei die Vortragende die deutsche, polnische und litauische Kulturerinnerung kontrastierte und dem Problem der Mythenbildung durch Ikonisierung besondere Aufmerksamkeit schenkte.

Die Sektion gab die Vielfalt der kulturellen Erinnerung insbesondere des 20. Jhd.s wieder, ihre Einteilung in Erinnerungen verschiedener Nationen und Kulturen, die Erinnerung der Opfer und der Täter, die deutsche, jüdische, polnische, litauische, kroatische Erinnerung sowie die Problematik der Kollektiverinnerung bei Roma und Sinti. Die Teilnehmer diskutierten u.a. über die Aktualität der in den literarischen Texten präsentierten kulturellen Erinnerungsräume, die Subjektivität/Objektivität der Erinnerung, ihren Wahrheitsstatus, ihre Bedeutung für die jeweilige Kultur, den subjektiven 
Charakter der Erinnerung der Geschichtswissenschaften, den Zusammenhang der individuellen und kollektiven Erinnerung, die intergenerationelle Erinnerung, die weibliche und männliche Erinnerung, die Mythisierung der Vergangenheit aufgrund der Erfahrung des Heimatverlustes, über die Bedeutung der Erinnerung für die Exilanten und über die Migrationsliteratur, schließlich über die Konstruierung der Vergangenheit im literarischen Text bzw. im Bild (im Film bzw. in der Buchillustration). Trotz der Globalisierung der heutigen Welt wurden durch die Ausführungen der Referenten die ganze Breite und der spezifische Charakter der nationalen, konfessionellen, gender- und generationsbedingten Erinnerung sowie ihr z.T. referentieller, z.T. individueller, subjektiver Charakter aufgezeigt.

Anna Dabrowska, Maria Kłańska,

Kraków

Sektion 21: Interkulturalität als Herausforderung und Forschungsparadigma der Literatur- und Medienwissenschaft (Leitung: Ortrud Gutjahr, Deniz Göktürk, Alexander Honold)

Die Sektion wurde mit einführenden Vorträgen der Sektionsleiter eröffnet. ORTRUD GUTJAHR (Hamburg) gab einen kritischen Überblick über neuere Diskussionen zu den Begriffen „Kultur“ und „Interkulturalität“" und zeigte die Notwendigkeit eines methodischen, kontextualisierenden und analytischen Diskurses auf, der sich mit der Essentialisierung von „Kulturen“ kritisch auseinandersetzt. Anschließend wies AlEXANDER HONOLD (Basel) darauf hin, dass die interkulturelle Literatur (wie die Reiseliteratur) interkulturelle Situationen nicht als „Beobachtung der Beobachtung", d.h. nicht von einer Metaebene aus wiedergibt, son- dern dass sie als Artefakt in ihren Narrativen Interkulturalität erst produziert. Zugespitzt plädierte DENIZ GÖKTÜRK (Berkeley) für „No-Culture“ bzw. für eine andere Semantisierung von „Kultur", die nicht in die Ethnisierung und Kulturalisierung von sozialen Problemen verfällt. In den darauf folgenden ca. 40 Vorträgen wurden verschiedene Aspekte, Themenschwerpunkte und Methodenvorschläge für Überlegungen zur „Interkulturalität als Forschungsparadigma“" präsentiert. DOERTE BISCHOFF (Hamburg) führte die Großstadt als Ort des heterogenen Miteinanders und als Schauplatz von Transkulturalität an Textbeispielen von Wladimir Kaminer, Doron Rabinovici und Yadé Kara vor. Die ,urbanen“ Romane von Yadé Kara wurden mehrfach besprochen: als literarische Inszenierung multikultureller Lebensläufe (MAHMUT KARAKUS, Istanbul) oder als neue literarische Darstellung des Umgangs mit (neuen Formen der) „Identität“ deutsch-türkischer Jugendlicher (VOLKER C. DÖRR, Bonn; KeIKo HamazaKI, Tokyo; GABRIELE EICHMANNS, Pittsburgh). Die interkulturelle Urbanität ist auch Thema eines Forschungsprojekts zu Prag als Topos der Interkulturalität, das MANFRED WEINBERG (Konstanz) vorstellte. Topoi der „Interkulturalität“ sind aber nicht nur vor urbaner Kulisse, sondern auch in der „tiefen Natur“ Afrikas zu beobachten. Die Problematik der literarischen Afrika-Darstellung, v.a. in Ilija Trojanows Weltensammler, wurde im Vergleich zu Urs Widmer und Uwe Timm von MichaEla HoldenRIED (Freiburg) und FRANZISKA BOMSKI (Freiburg) kritisch aufgegriffen. Angesprochen wurde außerdem die Intermedialität der Interkulturalität: KRISTINA FESTRING (Hamburg) interpretierte Yoko Tawadas Das nackte Auge intermedial mit Filmbeispie- 
len, und die filmische Darstellung der deutsch-polnischen „Interzone“ wurde von Randall Halle (Pittsburgh) auch im Hinblick auf transnationale Filmproduktionen vorgestellt.

Keiko Hamazaki, Tokyo

Sektion 22: Globalisierung - eine kulturelle Herausforderung für die Literaturwissenschaft? Germanistische Abgrenzungen (Leitung: Regina Hartmann, Klaus Bohnen, Bernd Neumann)

Den ersten Vormittag der Sitzung, die in dem schönen Senatssaal im Pałac Kazimierzowski stattfand, beherrschten eher theoretische Erwägungen, u.a. die Verbindung der Regionalität mit gesellschaftlichen Transformationen; die allmähliche Abkoppelung der Gesellschaft vom Raum, wodurch gegenwärtig die räumliche Definierung der Heimat aufgehoben wird, aber auch eine Wiederentdeckung des Heimatlichen und dessen neue Positionierung; der Zusammenhang zwischen Regionalität und Urbanität; Verlust der Heimat (der ehemaligen DDRBürger) und der Identität infolge der Hervorbringung sogenannter „Nicht-Orte“ (im Sinne von Marc Augé) durch die ,Übermoderne'. Im Mittelpunkt der Nachmittagssitzung standen zuerst Heimatund Migrationsproblematik, dies vor allem am Beispiel der türkisch-deutschen Geschichtsnarrative und Erinnerungskonstruktionen sowie in Werken deutschsprachiger Autoren mit den Wurzeln in der Sowjetunion bzw. Russland, in Polen, Rumänien oder Estland. Ein wichtiges Thema war dabei das Grenzgängertum zwischen den imaginären Sehnsuchtsorten der Kindheit und den realen Aufenthaltsorten. Den Rest des Nachmittags dominierte die Frage der Rezeption literarischer Texte über die deutschen
Grenzen hinaus (das Schaffen von Georg Büchner und Thomas Mann) sowie auch das Thema der Musik als Ausdruck nationalen Identitätsbewusstseins bei Thomas Mann.

Die Referate des zweiten Tages kreisten um konkrete Autoren und Orte, wobei der Einfluss der jeweils aktuellen Machtstrukturen hervorgehoben werden konnte. Es handelte sich um den sog. universellen Regionalismus bei den Deutschschweizer Autoren, dessen wichtige Bedingung die Weltoffenheit ist, um Geschichte als Geschichtetes und einen OrtDiskurs in Jenny Erpenbecks Roman Heimsuchung, um die ostpreußische Landschaft als Erinnerungsort, um die Rolle des Post- bzw. Transnationalen bei der Konstruktion der Identität sowie um die Palimpseststruktur einiger polnischer Städte, die als interkulturelle Begegnungsorte, Imaginationsräume oder Erinnerungsorte gedeutet werden können.

Die nach den Referaten folgenden Gespräche machten die Wichtigkeit der diskutierten Inhalte deutlich. Im Allgemeinen wurden in der Sektion interpretationsbedürftige Schwerpunkte hervorgehoben: Heimat und regionale Identität, die fortschreitende Ausdifferenzierung des Begriffs der Regionalität, Erinnerungsorte und Migration u.a. Selbstverständlich brachten die Diskussionen weder fertige Patentlösungen noch neue Definitionen, doch der internationale Gedankenaustausch sensibilisierte die Teilnehmer für andere Perspektiven. Nicht selten haben sich an den Diskussionen auch Mitglieder anderer Sektionen beteiligt, die - angeregt durch das Programmheft - an den Sitzungen teilgenommen haben.

Ewelina Kamińska, Dorota Sośnicka,

Szczecin 
Sektion 40: Deutsch-polnische Erinnerungsorte (Leitung: Leszek Żyliński, Sabine Doering, Elżbieta Dzikowska) Die Sektion griff die Thematik der deutsch-polnischen Erinnerungsorte aus verschiedenen interessanten Perspektiven auf. Einen methodologischen Einstieg in die Problematik der Erinnerungsorte gab KoRNELIA KoŃCZAŁ (PAN, Berlin) in ihrem Beitrag „Literatur als Medium des kollektiven Gedächtnisses: theoretische und methodologische Ansätze“. In zwei weiteren Vorträgen rückte die Thematik des Holocaust in den Vordergrund. GiSELA BRUdE-FIRNAU (Waterloo, Kanada) sprach über den Hunger im Ghetto Lodz/Litzmannstadt anhand der Chronik des Ghettos Lodz (2007). Die Referentin betonte, dass die Chronik die umfangreichste Dokumentation zum Holocaust aus der Perspektive der Opfer und heute ein bewegendes Medium der Erinnerung darstellt. Das Thema Holocaust nahm auch Charles Helmetag (USA) mit seinem Beitrag ,Erinnerungen ans Ghetto: Jakob der Lügner" in den Fokus seiner Überlegungen. In dem Vortrag wurde derselbe literarische Stoff in vier Versionen präsentiert: in dem Roman von Jurek Becker, im Kinderbuch und in zwei Verfilmungen. Damit eröffnete der Referent zugleich einen Einblick in die ästhetischkünstlerische Dimension des Begriffes ,Erinnerungsort', worauf Sabine Doering in der anschließenden Diskussion einging. Sie beklagte die Vernachlässigung dieser Dimension in der kulturwissenschaftlichen Gedächtnisforschung. Auf ein weiteres Defizit verwies Gisela Brude-Firnau, die das Postulat aufstellte, bei der Erforschung des kollektiven Gedächtnisses unbedingt den Begriff der ,historischen Treue' zu berücksichtigen. In der folgenden Referentengruppe lenkte Maria GierlaK (Toruń) den Blick auf verschiedene Facetten des Lebens der polnischen Putzfrau in der deutschsprachigen Gegenwartsliteratur. Auf das Berliner Konzept „Deutsch-polnische Erinnerungsorte " bezogen sich JERZY KAEĄŻNY (Poznań) mit dem Referat ,.,Grunwald und ,Teutoburger Wald' als parallele Erinnerungsorte?" und ElŻBIETA DZIKOWSKA (Łódź), die in ihrem Beitrag die Rezeption der Wanda-Figur in den Texten des 19. Jhd.s rekonstruierte. Dabei wurde in der folgenden Diskussion auf die internationale Dimension des Wanda-Mythos hingewiesen.

Ein historischer Aspekt der deutschpolnischen Erinnerungskultur kam in den Referaten der nächsten Referenten LESZEK ŻYLIŃSKI (Toruń) und BARBARA WIDAWSKA (Słupsk) zutage. Im Hinblick darauf tauchte in der anschließenden Diskussion die Frage auf, ob die polnische Putzfrau, das Datum 1968 oder die Person Wilhelms II. als Erinnerungsorte gefasst werden können. Es wurde aber auf die metaphorische Bedeutung des Noraschen Begriffes ,Erinnerungsorte hingewiesen, die ,nicht dank ihrer materiellen Gegenständlichkeit, sondern wegen ihrer symbolischen Funktion" zu Kristallisationspunkten kollektiver Erinnerung geworden sind. Demnach können also auch die in Frage gestellten Begriffe den Status der Erinnerungsorte beanspruchen (Sabine Doering). Hubert Lengauer plädierte jedoch für eine definitorische Begrenzung des Begriffes, Erinnerungsort". Er konstatierte, wenn man dieses Konzept metaphorisch so ausweite, dass jede Art von narrativem Komplex ein Erinnerungsort sei, dann gehe oft die spezifische Koppelung von Zeit und Raum verloren.

Die Referate der folgenden Gruppe beschäftigten sich mit literarisch gestalteten Erinnerungsorten. SABINE DOERING (Ol- 
Informationen und Berichte

denburg) zeigte in ihrem Beitrag, wie Thomas Manns Zauberberg zu einem Erinnerungsort in der polnischen $\mathrm{Ge}$ genwartsliteratur wurde. MARTINA KOLB (Yale University) beschäftigte sich in ihrem Vortrag „Teils-teils das Ganze: Gottfried Benns Nachkriegsmnemotopie“ mit dem poetischen Werk des Dichters, besonders im Zusammenhang mit dem zentralen Aspekt der Erinnerung an die Kindheit. Mit dem Stichwort „OderNeiße-Linie“ greift Benn im Gedicht Teils-Teils den Verlust der östlich der Oder gelegenen deutschen Gebiete und zugleich der Heimat seiner Kindheit auf. „Oder-Neiße-Linie für Sarginhalte ohne Belang" gebe hier vor allem Anlass zu kollektiven Vorstellungen der Erinnerung und $\mathrm{zu}$ einer genauen Betrachtung der Tiefendimension des Ortes als verräumlichter Zeit. ADOLF HÖFER (Deutschland) reflektierte in seiner Darstellung der Rezeption des Romans Die erste Polka von Horst Bienek die Bedeutung von Gleiwitz/Gliwice für die polnischdeutsche Geschichte. Er betonte, dass diese Heimat zwar für die Deutschen für immer verlorengegangen, im Gedächtnis der Dichtung aber bewahrt geblieben sei. Die Hoffnung auf die literarische ,Wiedergewinnung' der alten Heimat sollte das neue Verhältnis zu den Nachbarn im Osten bestimmen und damit einen wichtigen Beitrag zur Versöhnung und zum friedvollen Zusammenleben der Völker leisten. Sigfrid HoEFERT (Kanada) referierte über den Roman Die Friedensinsel und über das zum thematischen Umfeld des Romans gehörende Hörspiel Richelieu entdeckt Danzig von Max Halbe, der den deutsch-polnischen Konflikt in Danzig thematisierte. In der anschließenden Diskussion wurde darauf hingewiesen, dass man ein wenig kritischer betrachten sollte, wie sich Halbe, der sich von den
Nazis benutzen ließ, in diesem Konflikt positionierte. Zusammenfassend lässt sich feststellen, dass sich in den Diskussionen als recht problematisch vor allem der Begriff ,Erinnerungsort " erwiesen hat. Dennoch haben die Referate und Diskussionen einen wichtigen Beitrag zur Annäherung an die Thematik geleistet und eine Reihe von nationalen und binationalen Erinnerungsorten thematisiert.

Barbara Widawska, Słupsk

Sektion 41: Jiddische Sprache und Literatur in Geschichte und Gegenwart (Leitung: Simon Neuberg, Steffen Krogh, Ewa Geller)

Seit dem VII. Internationalen Germanistenkongress in Göttingen hat die Jiddistik im Rahmen der Germanistik ihren festen Platz gefunden. Auf dem diesjährigen Kongress in Warschau haben an der Jiddistiksektion leider nur vierzehn Wissenschaftler, dafür aber aus aller Welt teilgenommen: Außer aus Europa gab es Forscher aus Russland, China und Nordamerika. Dabei wurden verschiedene Themen der modernen Jiddischforschung angesprochen. Präsentiert wurden linguistische sowie literatur- und kulturwissenschaftliche Forschungsberichte.

Die einzelnen Referate wurden auf vier thematische Sitzungen verteilt, die den Tagungsablauf organisiert haben. Allerdings hat das Thema der gegenseitigen Beziehungen zwischen dem Deutschen und dem Jiddischen die Sektionstagung des diesjährigen Kongresses dominiert. Dieses Problem kehrte immer wieder nicht nur in den einzelnen Referaten, sondern auch in den Gesprächen zwischen den einzelnen Teilnehmern. In Einzelreferaten mit unterschiedlichen Ansätzen wurden die Einflüsse des Deutschen auf die jiddische Literatur und 
Sprache diskutiert. In diesem Sinne wurden z.B. die Dajtschmerismen, die Wörter, die durch die Sprecher des Jiddischen als Entlehnungen aus dem Deutschen eingestuft werden, zum Leitmotiv der Sektionssitzung. Auch die umgekehrte Richtung der Einflussnahme stand im Interessenbereich der Teilnehmer der Jiddistiksektion. So wurde der Einfluss des Polnischen auf die Entstehung und Weiterentwicklung des Jiddischen unter die Lupe genommen. Inwieweit die Jiddischsprecher und -schreiber auf der einen Seite aus dem Deutschen geschöpft haben und auf der anderen gegen den deutschen Einfluss vorgegangen sind, wurde eingehend diskutiert. Ein wichtiger Teil der Referate konzentrierte sich auch auf autobiographische Motive und biographische Erfahrungen, die sich in der jiddischen Literatur, Sprache und Kultur widerspiegeln. Man beschäftigte sich u.a. mit autobiographisch gefärbten Darstellungen des polnischen Judentums oder mit den widersprüchlichen Aussagen über die eigene Biographie mancher jiddischer Schriftsteller. Nicht zuletzt soll erwähnt werden, dass einige Probleme der historischen jiddischen Sprachforschung aufgegriffen und neuere Erkenntnisse auf diesem Gebiet präsentiert wurden.

Zusammenfassend ist festzuhalten, dass die Abwesenheit mancher ausgewiesener Forscher sowie das durchaus unterschiedliche Niveau der Referate Fragen über die Zukunft einer weitverstandenen Jiddistik im Rahmen der Germanistik aufwerfen. Vielmehr sollte Jiddistik heutzutage als ein die traditionellen Grenzen der Germanistik übergreifendes Gebiet verstanden werden.

Agata Kondrat, Warszawa
Sektion 2: Textsorten und Sprachgeschichte (Leitung: Józef Wiktorowicz, Jörg Meier, Arne Ziegler)

Die Diskussionen konzentrierten sich auf die Fragen der historischen Entwicklung von Textsorten vom 16. bis zum 20. Jhd. Einige Beiträger besprachen einzelne grammatische Erscheinungen in einer Textsorte bzw. in Texten einer Epoche. Weitere Vorträge wurden Aspekten der textlinguistischen Analyse und ihrer Nutzung bei historischen Untersuchungen gewidmet.

Sektion 11: Beschreibende deutsche Grammatik (Leitung: Józef Darski, John Ole Askedal, Akio Ogawa, Irina Schipowa)

Im Mittelpunkt des Interesses befanden sich unterschiedliche grammatische Beschreibungsmodelle der deutschen Sprache. Die einzelnen Redner unterbreiteten entweder ihre Konzepte einer Grammatik der deutschen Sprache für unterschiedliche Adressaten (z.B. wissenschaftliche Grammatik, Schulgrammatik) oder erörterten ausgewählte Aspekte der deutschen Gegenwartssprache und wiesen auf die Möglichkeiten ihrer Behandlung in einer Grammatik hin. Es wurde auch am Rande auf einige Fragen des Sprachvergleichs (Deutsch - Koreanisch) eingegangen.

Sektion 24: Germanistische Textlinguistik (Leitung: Margot Heinemann, Zofia Bilut-Homplewicz, Marianne Hepp)

Eine ganze Reihe von Analysen ausgewählter Textsorten wurden hier in den Mittelpunkt gestellt. Es war eine der größten Sektionen in Warschau mit über 30 gehaltenen Vorträgen. Neben den empirischen Studien wurde auch mehrmals versucht, die bisherigen Textanalysemo- 
delle auf den Prüfstein zu stellen und sie $\mathrm{zu}$ modifizieren. Außerdem wurde das Verhältnis zwischen der Textlinguistik und der Diskursanalyse angesprochen. Dank einiger Referate lie $\beta$ sich eine interessante Tendenz beobachten, nämlich der Versuch, die Ergebnisse der Textlinguistik auf $\mathrm{DaF}$ anzuwenden und sie für den Deutschunterricht nützlich zu machen.

Sektion 30: Deutsche Dialekte und Regionalsprachen (Leitung: Ewa Żebrowska, Elvira Glaser)

In der Diskussion wurde die Vielfalt der deutschen Sprache thematisiert. Intensiv wurden einzelne Phänomene des Schweizerdeutschen behandelt. Einen anderen Schwerpunkt der Referate und Diskussionen stellten neue Entwicklungstendenzen der deutschen Dialekte (z.B. das Niederdeutsche im urbanen Raum) dar.

Sektion 39: Digitalität und Textkulturen (Leitung: Beata Mikołajczyk, Henning Lobin)

Diese Sektion befasste sich mit der zentralen Frage, inwieweit unsere durch das Buchwissen geprägten Kulturen durch den Computer, das Internet, durch TV sowie die damit verbundene neue Bildlichkeit nachhaltig verändert werden. $\mathrm{Zu}$ den Schwerpunkten dieser Sektion gehörten in erster Linie zwei Themenbereiche, 1. der Einfluss der digitalen Medien auf das Sprachverhalten und den Textbegriff, 2. der Einfluss der sog. Neuen Medien auf die Gewohnheiten der Menschen bei der Textproduktion und -rezeption, u. a. die Entstehung neuer Textsorten und Kommunikationsformen und die Modifizierung der , alten'. Neben diesen allgemeinen Fragen standen auch einzelne Textsorten (z. B. Chat, SMS, wissenschaftliche Rezension im Internet, wissenschaftliche Präsentation) zur Debatte. Beata Mikotajczyk, Poznań
Für die in dieser Auswahl nicht berücksichtigten Sektionen sei auf die zum Kongress erschienene Publikation der Abstracts verwiesen. ${ }^{1}$ Die Plenarveranstaltungen beschäftigten sich vorwiegend mit den Themen Interkulturalität und Transkulturalität sowie mit der Frage nach der Zukunft der Germanistik und der Attraktivität der deutschen Sprache und Literatur im Ausland, es fand aber auch je ein Panel zur Literatur der Vormoderne und zu Regionalsprachen statt. Wie auf der gesamten Tagung kam es auch hier zu Überschneidungen. Dass die Organisatoren anders als bei den vorhergehenden Kongressen auf Plenarreferate so gut wie ganz verzichtet hatten, wurde von nicht wenigen Teilnehmern als bedauerlich empfunden.

Unter den Veranstaltungen des Rahmenprogramms sei neben einem beeindruckenden Liederabend mit Ruth Ziesak, die Lieder von Schumann, Liszt und Mahler interpretierte, die Verleihung des Jakob- und Wilhelm-Grimm-Preises an den amerikanischen Germanisten DAVID E. WELLBERY hervorgehoben. Er sprach in seiner Dankesrede über ,Die Dramatisierung des Schönen“ in Goethes Pando$r a$ und las in ihr Prometheus und Epimetheus als zwei Figuren der modernen Bewusstseinsentzweiung.

Der Kongress fand in einer nicht nur meteorologisch, sondern auch erinnerungspolitisch heißen Zeit statt. Wer wollte, konnte an den Feierlichkeiten zum Beginn des Warschauer Aufstandes am 1. August teilnehmen, und wohl kaum jemand ging unbewegt am Kreuz vor dem Präsidentenpalast vorbei, das an den Absturz des polnischen Regierungsflugzeuges in Smolensk am 10. April 2010 erinnerte. Die Teilnehmerinnen und Teilnehmer des Kongresses hatten die Möglichkeit, an Stadtführungen und an 
Ausflügen, u.a. nach Krakau und Danzig, teilzunehmen. Da diese Angebote aber überteuert waren, wurden sie nur von wenigen genutzt. Einige Gäste begaben sich lieber privat auf eine Reise durch das Land. Die ebenfalls überhöhten Übernachtungskosten waren sicher eine der Ursachen dafür, dass so manch ein Referent nicht anreiste bzw. früh wieder abreiste und dass relativ viele Vorträge ausfielen. An der Vollversammlung am letzten Kongresstag nahmen knapp 300 Germanisten teil, weshalb der Vorschlag unterbreitet wurde, die Versammlung auf dem nächsten Kongress in die Mitte des Tagungszeitraums zu legen. Für das Präsidentenamt kandidierten Paul Michael Lützeler (St. Louis) und Jianhua Zhu (Shanghai). Letzterer wurde zum neuen Präsidenten gewählt. Damit wird der IVG-Kongress im Jahr 2015 in China stattfinden. Als Vizepräsidenten wurden
Laura Autri (Palermo) und Adjaï A. Paulin Oloukpona-Yinnon (Lomé) gewählt. Trotz mancher Mängel in der Organisation der Konferenz, zu denen auch das Nichtbereitstellen eines Mitgliederverzeichnisses gehörte (laut Statut des IVG, Art. 7, Abt. 6, hätten die Mitglieder ein solches Verzeichnis erhalten müssen), gebührt den Organisatoren Dank für ihr Engagement, durch das die in Warschau angereisten Gäste die Möglichkeit erhielten, unmittelbare Einblicke in die internationale germanistische Forschung $\mathrm{zu}$ gewinnen, die sich gewiss in Inspirationen für ihre eigene wissenschaftliche $\mathrm{Be}$ tätigung niederschlagen werden.

\section{Anmerkung:}

${ }^{1}$ Internationale Vereinigung für Germanistik. XII Kongress Vielheit und Einheit der Germanistik weltweit. Abstracts. Universität Warschau 30.07.-7.08.2010.

Marion Brandt, Gdańsk 